\title{
Dynamic Testing of an IEC 61850 Based 110 kV Smart Substation Solution
}

\author{
Jiancheng Tan, Shuxian Zhang, Jun Mo, Xiaoping Xiong, Bilian Liao, Chao Zhang \\ Smart Substation Technologies and Applications Lab, Guangxi University, Nanning China \\ Email: jctan@gxu.edu.cn, mojin1232@sina.com, xxp001@sina.com, lbsjx519@163.com
}

Received August 2014

\begin{abstract}
This paper presents the dynamic simulation and testing to verify the smart substation solutions designed for a brown field $110 \mathrm{kV}$ retrofitting project. An IEC 61850 based aotomation design, transitioning the conventional substation into a smart substation, where existing current/voltage transformers remain in service, and smart Field Apparatus Interface Units (FAIUs) are utilised to bridge the conventional primary system to the IEC 61850 based secondary system. While outdoor switchgears and field instrument transformers are equipped with FAIUs, MV indoor switchgears are installed with IEDs mounted on the top. Direct point-to-point connections serve as process buses, and a single PRP/RSTP LAN is employed at station bus level. Extensive dynamic simulation and testing were conducted in the Smart Substation Technologies Lab, and test results show the smart substation performance meets and exceeds the substation reliability requirement.
\end{abstract}

\section{Keywords}

Process Bus, Station Bus, GO0SE, Digital Substations, Smart Substations, Parallel Redudancy

\section{Introduction}

At present, China is undergoing the development of a large-scale strong solid power grid. The complexities of power systems associated with various operational modes, demands smart and intelligent apparatus, and more efficient information exchange at all levels. New substations should be built in a new way, in order to facilitate easy engineering, shorten installation and commissioning time, and be capable of performing incremental upgrades in the future in a flexible and robust way. The new national-wide initiative for smart substations aims at tapping to the latest technology available for innovative solutions [1] [2].

The global standard IEC 61850 facilitates seamless information exchange between apparatus and the network based secondary system. Moving towards an information highway for protection, control, automation and beyond, opens up a world of opportunities in engineering, construction, commissioning, operation and maintenance. With the ample proof of its significant savings incurred in thousands projects world-wide [3]-[5], IEC 61850 based smart substation solutions are proven to be viable and cost effective [6]-[8].

This paper investigates an IEC 61850 related smart substation solution, where intelligent field interface units (FAIUs) are employed to transfer conventional current/voltage transformers and outdoor switchgears into smart 
apparatus. The FAIUs are of merging unit modules, integrated with RTU and communication functions, which acquires the analog current and voltage signals, digitizes them into IEC 61850-9-2LE sampled value streams, and transmits them over the dedicated point-to-point connected process buses. The field apparatus interface units (FAIUs) are of rugged modular design, configurable Sampled Value (SV) and GOOSE datasets, supports up to eight 100mbps FX ports, thus are flexible, robust and versatile for field deployment. At the station bus level, a single substation LAN is designed for protection signaling, SCADA control and remote access, where parallel redundancy protocol (PRP) and rapid spinning tree protocol (RSTP) are used to ensure reliable data transmission with guaranteed delivery of mission critical and time stringent protection signals.

The $110 \mathrm{kV} / 10 \mathrm{kV}$ smart substation demonstrates the use of FAIUs for sampled value streams over the proposed process bus, and for GOOSE applications over the substation LANs. A complete secondary system is constructed in the Smart Substation Technologies Lab, Guangxi University, where Real Time Digital Simulator (RTDS) is used to model the primary system of the substation equipment. Current and voltage signals from the relaying points from RTDS are amplified to the true secondary level and injected onto the FAIUs and relays under testing, with statuses of indoor and outdoor switchgears from the RTDS hard wired to the FAIUs and relays. Decisions from the secondary system, i.e. either the FAIUs or relays are fed back to the RTDS, and the late continues simulating the power system in real time until it reaches a new steady state or have lost stability. A RTDS batch test program for automatic testing and recoding is developed in the lab, which repeats the same test for a pre-set 100 times. While during the tests, a traffic generation device hosted in the engineering station generates network traffics and injects them into the station bus LAN, to flood the substation communication infrastructure. Test results were plotted to verify the proposed solutions under various background traffic conditions.

Extensive tests were conducted in the lab. Analyzing the recorded test results, no package drops were observed. Sampled value streams and GOOSE tripping over the LAN is proven to be fast, reliable and not affected by the background traffic over the proposed substation LAN, where dedicated point-to-point connections are utilized at process bus level, which contain the process bus traffic into where absolutely required, and a single PRP/RSTP LAN for multicasting GOOSE messages, SCADA applications and remote access etc, and is proven to be robust and reliable.

\section{Overview of $110 \mathrm{kV} / 10 \mathrm{kV}$ Substations}

The $110 \mathrm{kV} / 10 \mathrm{kV}$ substation is of eight indoor switchgear feeders connected onto two separated busbars. The two bus sections are connected via a normally closed bus sectionaliser. A transfer bus with a bus coupler dedicated for maintenance related tasks is installed but not shown in Figure 1. While the $10 \mathrm{kV}$ indoor switchgear

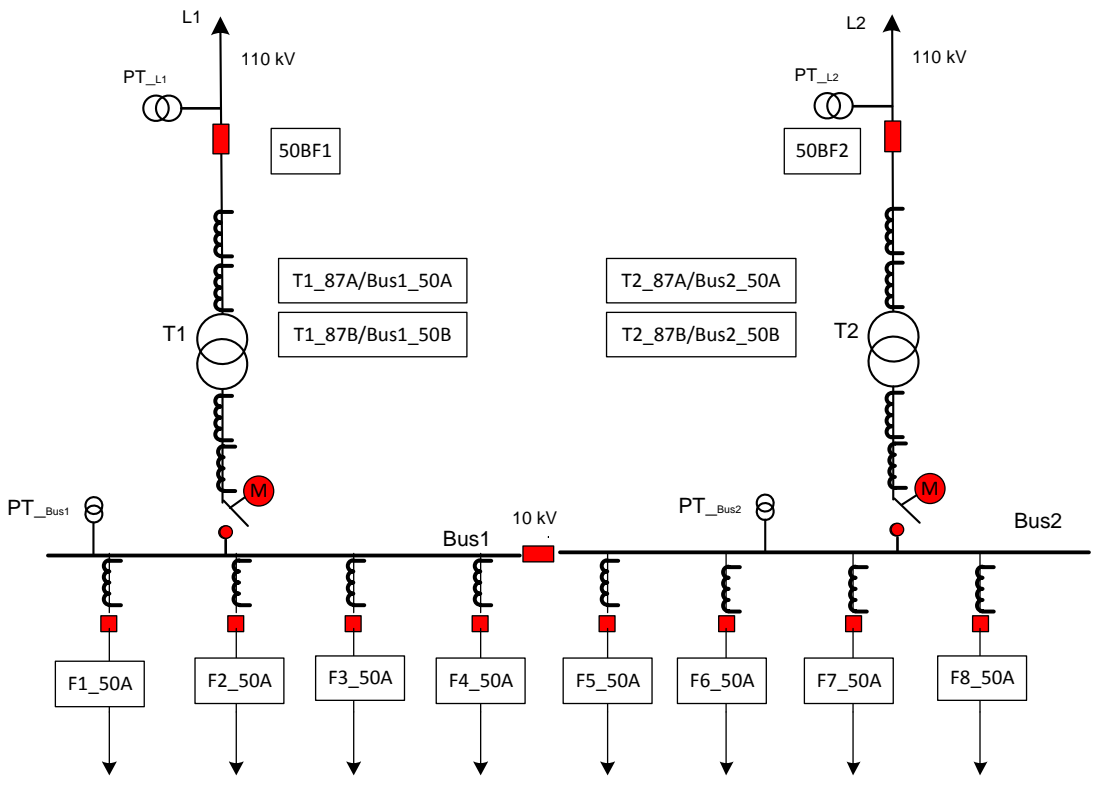

Figure 1. $110 \mathrm{kV} / 10 \mathrm{kV}$ substation arrangement. 
system is within the control building, the $110 \mathrm{kV}$ lines and transformers are outdoor in the switchyard. Each 10 $\mathrm{kV}$ bus section is supplied by a $110 \mathrm{kV} / 10 \mathrm{kV}, 25$ MVA transformer with a two (2) cycle breaker on the HV side. There is no circuit breaker but a motor driven disconnect switch at the $10 \mathrm{kV}$ side.

Distributed generations (DGs) are tapped onto the $10 \mathrm{kV}$ feeders. As observed in Figure 1, if a fault occurs on $\mathrm{T} 1$, or on Bus1, fault isolation requires tripping the associated HV breaker, the bus sectionaliser, and all feeder breakers connected on Bus 1. Thus, integrated dual redundant transformer and bus protection schemes are applied, and each $110 \mathrm{kV}$ breaker failure protection is a standalone single breaker failure (50BF) relay hard wired from all digital and non-didgital relays tripping the breaker. Upon detection of a breaker failure, the 50BF will initiate the trip to all electrically connected breakers, as well issue direct transfer trip (DTT) signals to the remote substations where lines are directly connected with the fauty breaker. No $110 \mathrm{kV}$ line protection is applied in this case.

\subsection{0 kV Feeder Protection}

A feeder relay provides feeder protection and the associated controls, is configured to receive GOOSE trip signals from the $110 \mathrm{kV}$ 50BF relay and the transformer/bus relays to trip the feeder breaker upon a breaker failure, transformer or bus fault.

The feeder relay will issue a GOOSE signal to the associated bus relay upon detection of a fault, a motor start up or load encroachment condition. The feeder relay will also issue a GOOSE trip to the Bus relay if a breaker failure condition is detected. The following GOOSE publications are applied

- Feeder fault GOOSE Blocking;

- Feeder downstream motor start-up GOOSE Blocking;

- Feeder load encroachments GOOSE Blocking;

- Feeder breaker failure Bus GOOSE Trip.

\section{2. $10 \mathrm{kV}$ Bus Protection}

Medium Voltage Switchgear 10kV Bus protection is designed with a fast bus protection scheme, and a coordinated time overcurrent scheme in parallel. The bus relay trip decision is delayed for a pre-set four (4) cycle delay in the fast bus scheme, and a minimum $300 \mathrm{~ms}$ delay time in the coordinated overcurrent scheme. The fast bus protection scheme was a hard wired approach, simple in principle, easy to expand, but requires information exchange between multiple devices. The complicated hard-wiring is error-prone, and tremendously increases the installation and maintenance costs. In this application, wiring between the bus and other relays are removed, and GOOSE messages are employed, with the blocking time delay reduced to two (2) cycles. Upon a Bus fault, a GOOSE BF Initiation will be issued to the 50BF relay, and reclose of the feeder breaker is prohibited. While the hard wired solution requires two seperated connections, a single multicasting GOOSE Bus Trip signal is sufficient to serve all.

The following GOOSE messages are configured to receive:

- Feeder 1 Fault/Start-up/Load Encroachment GOOSE Blocking;

- Feeder 2 Fault/Start-up/Load Encroachment GOOSE Blocking;

- Feeder 3 Fault/Start-up/Load Encroachment GOOSE Blocking;

- Feeder 4 Fault/Start-up/Load Encroachment GOOSE Blocking;

- Feeder 1 Breaker failure GOOSE Bus Trip;

- Feeder 2 Breaker failure GOOSE Bus Trip;

- Feeder 3 Breaker failure GOOSE Bus Trip;

- Feeder 4 Breaker failure GOOSE Bus Trip.

\subsection{Transformer Protection}

Protection of the $110 \mathrm{kV} / 10 \mathrm{kV}$ transformers is provided by dual differential schemes. Upon detection of a transformer fault, a GOOSE BF initiation signal is issued, and cancel reclose GOOSE message is send to prevent the breaker being reclosed. The following GOOSE messages will be published:

- Transformer fault GOOSE BF Initiation;

- Transformer fault GOOSE cancel recluse; 


\subsection{BF Protection}

The breaker failure 50BF relay will issue GOOSE BF trips to open the $10 \mathrm{kV}$ feeder breakers upon detection of a breaker failure condition, as well sends direct transfer trip signals to the remote line ends relays. The 50BF relay receives the following BF initiation signals from digital relays:

- Transformer/Bus A relay GOOSE BF Initiation;

- Transformer/Bus B relay GOOSE BF Initiation;

- Non-digital relay hard wired Initiation.

All digital relays are configured to receive the associated switchgear GOOSE status messages, and report to the Gateway/HMI for SCADA applications. Station wide controls are implemented using the HMI single line diagram graphic interface.

For outdoor switchgears, field apparatus interface units/boxes (FAIUs) are used to transfer the conventional current and voltage transformers and circuit breakers into smart apparatus.

\section{Field Apparatus interface Units}

The field apparatus interface unit (FAIU) serves as the interface units between primary systems in the switchyard and secondary systems in the control building. As illustrated in Figure 2, the FAIU units are equipment with two analog modules, hard wired to conventional current and voltage transformers, as well switchgears such as breakers and disconnect switches in the switchyard. Connections to the secondary IEDs in the control building are via optical fiber connections. The functional modules of the FAIUs are as below:

\subsection{Merging Unit Functions}

A merging unit module may equip two analog modules, acquires analog current and voltage signals, and digitizes them into maximum 4 sets of sampled value streams according to IEC 61850-9-2LE. Each set of the sampled value streams is configurable with $(4 \mathrm{I}, 4 \mathrm{~V})$ or $(4 \mathrm{I}, 4 \mathrm{I})$ signals, and is independently assigned with the communication ports specified. The following analog modules are available in a FAIU:

- $4 \mathrm{I}(5 \mathrm{~A}), 4 \mathrm{~V}$;

- $4 \mathrm{I}(1 \mathrm{~A}), 4 \mathrm{~V}$;

- 8I (5A);

- $8 \mathrm{I}(1 \mathrm{~A})$.

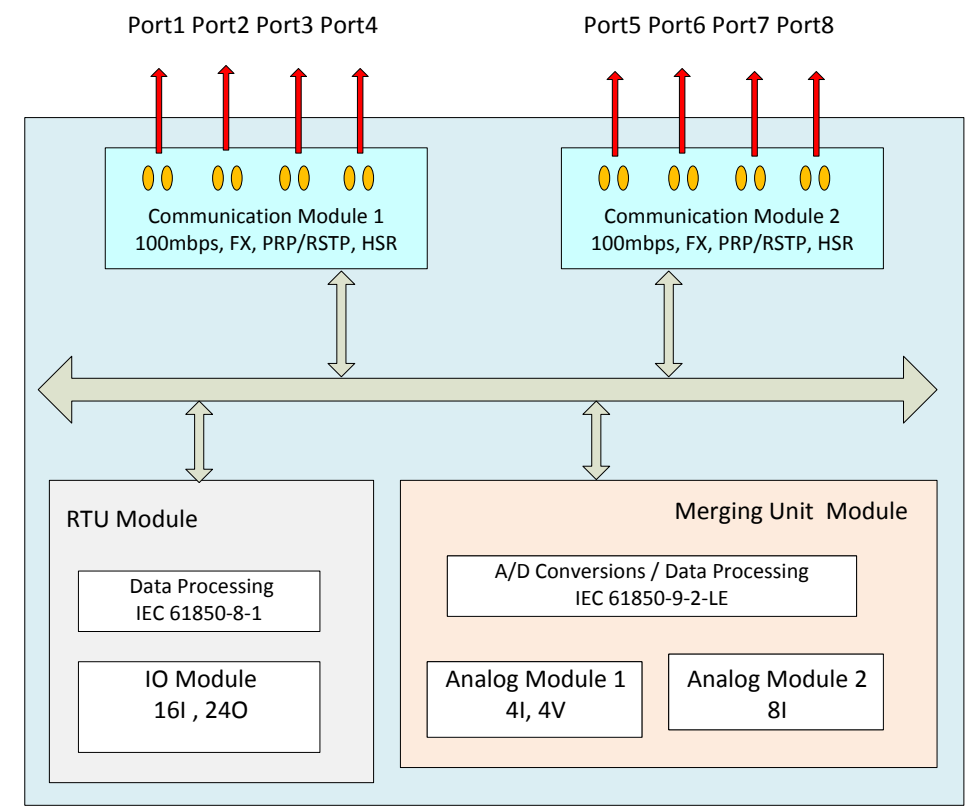

Figure 2. Field interface unit t. 


\subsection{Remote Terminal Unit Functions}

A Remote Terminal Unit module interfaces with switchyard circuit breakers and disconnect switches, is of the bay control unit function. It acquires the breaker status, processes them as various GOOSE data sets according to IEC 61850-8-1 and publish tem onto the station bus LAN. Each GOOSE data sets can be configured individually and can be assigned to specific ports.

\subsection{Communication Module}

Each communication module is of 4 ports, each port is capable of transmitting the assigned sampled value streams, and/or combined with GOOSE message transmission, as well receiving GOOSE messages, support PRP, HSR and/or RSTP redundancy protocols.

The configurable field interface units (FAIUs) are robust, flexible and scalable, serve as key elements in green field substations installed with conventional CTs/PTs, or for substation retrofitting solutions, where conventional apparatus installed have an average life cycle of 60 years.

\section{Substation Architecture}

The reliability of the substation architecture is vital to the acceptance of the industry. This is particularly true to field deployment when time stringent and mission critical protection signals such as sampled values and trip signals come to play. The reliability of the process bus system not only depends on the merging units and the process bus relays utilised, the process bus architecture and the associated redundancy protocols supported by the LAN and the IEDs, will also have significant impact, as well the volume of traffic and their related priority levels while transmitting on the network.

\subsection{Process Bus}

Considering an IEC61850-9-2LE sampled value data stream consuming approximate 5mbps bandwidth, at system frequency $50 \mathrm{~Hz}$ and the defined sampling rate of 80 samples per cycle, process bus traffic is of high volume in nature, and consistent all time, thus must be carefully engineered at the process bus level, and bridge to where absolutely needed, and provide means of isolation from the mission critical and time stringent protection tripping signals.

Table 1 illustrates the detailed current and voltage configuration and assignments. In this application, dual redundant field interface units are employed for the transformer/bus protection schemes. Six (6) process bus relays and four (4) field interface units are designated for process bus applications. The protection relays include:

- T1_87A/Bus1_50A

- T1_87B/Bus1_50B

- 50BF1

- T2_87A/Bus2_50A

- T2_87B/Bus2_50B

- $50 \mathrm{BF} 2$

The field interface units are hard wired to acquire the current and voltage signals, as well the status information circuit breakers and disconnect switches, and are of the following modules:

- Module 1: Merging Unit module;

- Module 2: RTU module;

- Module 3: Communication module.

Where, the Merging Unit module consists of two (2) 4I, 4V analog modules, and the copper connections with the current, voltage transformers and outdoor switchgears are as described in Figure 3.

The field interface unit is configured to transmit various sampled value streams for specific protection applications. A transformer/bus relay will receive sampled values from one single field interface unit, information received include: 
Table 1. IEC 61850-9-2le sampled value datasets assignments.

\begin{tabular}{ccccccccc}
\hline SV Dataset & \multicolumn{1}{c}{4} & I & & 4 & V \\
\hline SVT1_11 & CT_T1H1A & CT_T1H1B & CT_T1H1C & CT_T1H1G & PT_L1A & PT_L1B & PT_L1C & PT_L1G \\
SVT1_12 & CT_T1L1A & CT_T1L1B & CT_T1L1C & CT_T1L1G & PT_Bus1A & PT_Bus1B & PT_Bus1C & PT_Bus1G \\
SVT1_21 & CT_T1H2A & CT_T1H2B & CT_T1H2C & CT_T1H2G & PT_L1A & PT_L1B & PT_L1C & PT_L1G \\
SVT1_22 & CT_T1L2A & CT_T1L2B & CT_T1L2C & CT_T1L2G & PT_Bus1A & PT_Bus1B & PT_Bus1C & PT_Bus1G \\
SVT2_11 & CT_T2H1A & CT_T2H1B & CT_T2H1C & CT_T2H1G & PT_L2A & PT_L2B & PT_L2C & PT_L2G \\
SVT2_12 & CT_T2L1A & CT_T2L1B & CT_T2L1C & CT_T2L1G & PT_Bus2A & PT_Bus2B & PT_Bus2C & PT_Bus2G \\
SVT2_21 & CT_T2H2A & CT_T2H2B & CT_T2H2C & CT_T2H2G & PT_L2A & PT_L2B & PT_L2C & PT_L2G \\
SVT2_22 & CT_T2L2A & CT_T2L2B & CT_T2L2C & CT_T2L2G & PT_Bus2A & PT_Bus2B & PT_Bus2C & PT_Bus2G \\
\hline
\end{tabular}

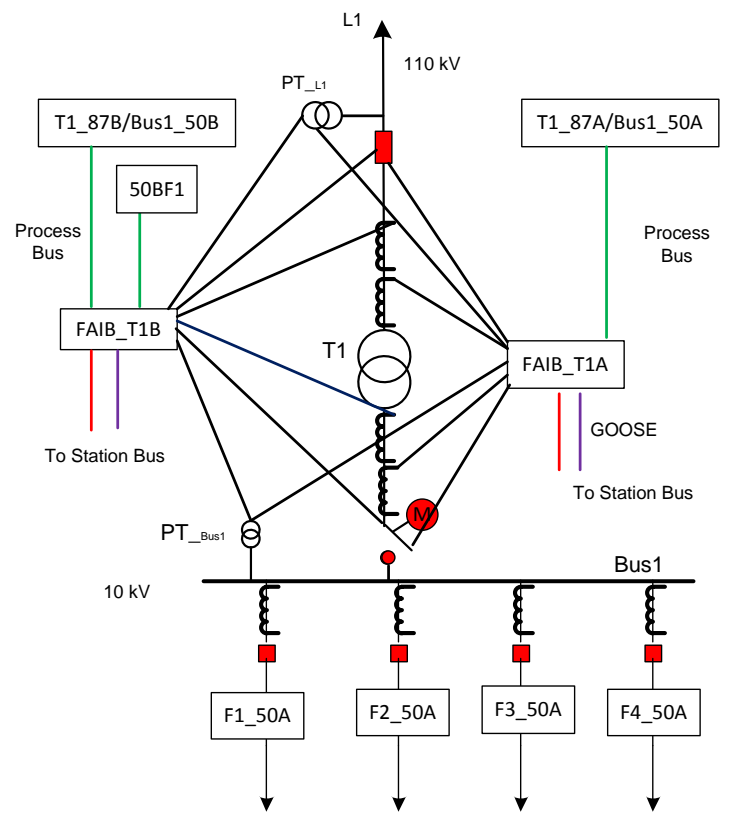

Figure 3. Process bus (partial).

- Four (4) Currents from transformer HV bushing CT;

- Four (4) Currents from transformer LV bushing CT;

- Four (4) Voltages from $110 \mathrm{kV}$ Line PT;

- Four (4) Voltages from $10 \mathrm{kV}$ Bus PT.

As demonstrated in Table 2, a field interface unit also supplies current and voltage signals to the breaker failure relay. No redundancy is required for standalone breaker failure protection.

Data transmitted over a process bus is mission critical. In this application, point-to-point fiber connections from field interface units (FAIUs) to relays are utilized as process bus architecture, this contains process bus traffic to where it is absolutely required. This simple architecture design ensures short latency delay, prevents traffic flooding the LAN. It is simple, robust, easy to maintain, and reliable to operate. The scalability and expandability of the overall substation process bus applications are inherently achieved by the field interface units deployment strategies, thus are flexible, robust and versatile for all substation configurations.

\subsection{Station Bus}

Previous applications for station bus architecture design utilizes rugged Ethernet switches and rapid spanning tree protocol (RSTP) as self-healing redundancy mechanism. While it is proven to be viable with many industrial applications, it is doubtful that reliability of the Protection/SCADA system may subject to issues, and there 
is possibility reliability of the system may decline due to complicated routing algorithms and configurations, which in return may probes more human error during engineering and maintenance stages.

1) Ethernet Switch

Two substation grade Ethernet switches are installed to form up the parallel redundancy network for station bus, each with $24 \times 100$ mbps FX ports, $4 \times$ RJ45 100/1000 mbps ports for maintenance and engineering access.

2) Parallel Redundancy Protocol

All IEDs and FAIUs in the secondary system supports PRP/RSTP redundancy, this gurantees no GOOSE messsage drops, for network architecture design as depicted in Figure 4.

3) VLAN and Priority Tagging

No VLANs are applied. VLAN ID is set to zero. Priority mapping between IEC 61850 and Ethernet Switch hardware related priority forward is given in Figure $\mathbf{5}$.

4) Message Priority Assignments

All GOOSE messages transmitted over the station bus LAN are with priority tagging, and are transmitted over the LAN, potentially available to all IED connected. The priority of GOOSE message assignments are based on the signal missions inherited, and are listed in Table 3.

All GOOSE messages transmitted over the Station LAN are priority tagged. Other traffic such as SCADA MMS alarms, remote access, file transfers etc. are dealt with best effort.

The Station Bus LAN employs PRP/RSTP redundancy, requires all IEDs connected support the PRP protocol. Minimum switch configuration is required, simple, easy and straightforward.

\section{Solution Evaluation}

A fully functional secondary system with all protective relays, substation Gateway to SCADA, and HMI for alarm processing and station wide control of the $110 \mathrm{kV} / 10 \mathrm{kV}$ substation as depicted in Figure 1 has been developed and constructed in the Smart Substation Technology LAB of Guangxi University.

The real time digital simulator (RTDS) models the detailed apparatus including the two $110 \mathrm{kV}$ transmission lines transformers, circuit breakers, current and voltage transformers etc. in the $110 \mathrm{kV} / 10 \mathrm{kV}$ substation, and grid beyond the two $110 \mathrm{kV}$ lines are modeled using simplified grid models and equivalent sources at both ends.

Table 2. Field interface units assignments.

\begin{tabular}{cccc}
\hline Protection & SV1 & SV2 & FAIU \\
\hline T1_87A/Bus1_50A & SVT1_11 & SVT1_12 & FAIU_T1A \\
T1_87B/Bus1_50B & SVT1_21 & SVT1_22 & FAIU_T1B \\
50BF1 & SVT1_21 & & FAIU_T1B \\
T2_87A/Bus2_50A & SVT2_11 & SVT2_12 & FAIU_T2A \\
T2_87B/Bus2_50B & SVT2_21 & SVT2_22 & FAIU_T2B \\
50BF2 & SVT2_21 & & FAIU_T2B \\
\hline
\end{tabular}

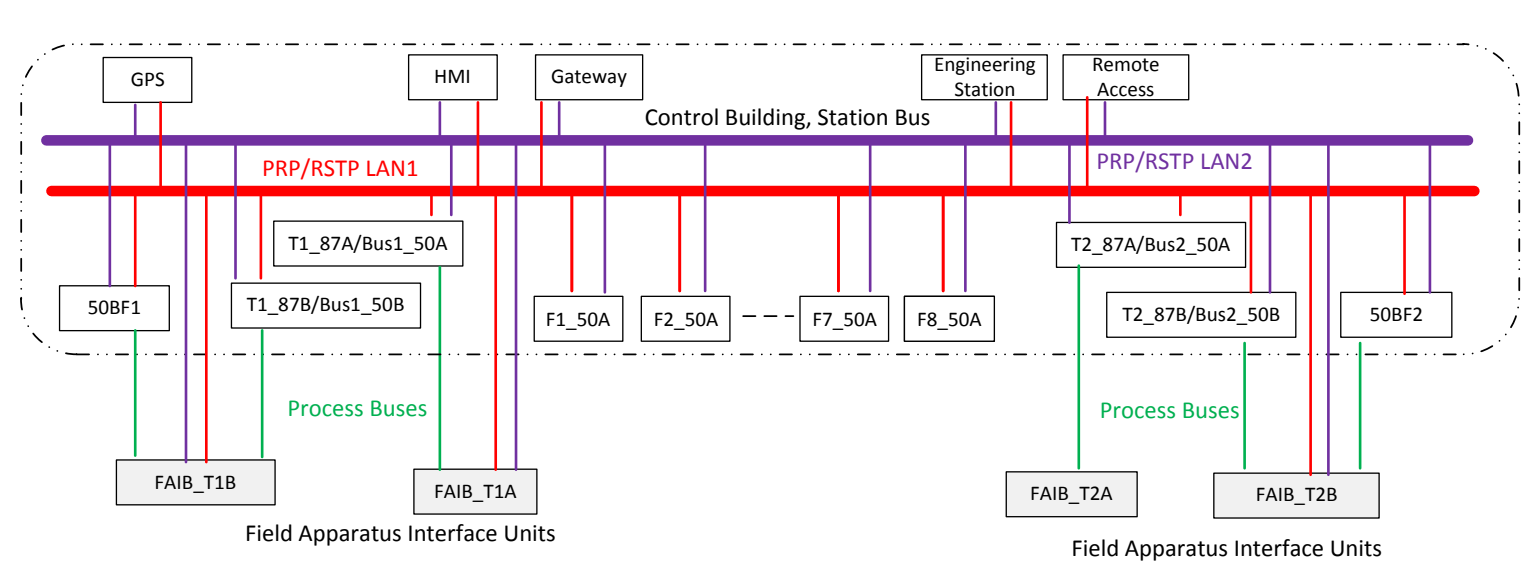

Figure 4. Substation LAN Architecture. 


\begin{tabular}{|c|c|c|}
\hline IEC 61850 & Priority Mapping & $\begin{array}{c}\text { Ethernet } \\
\text { Switch }\end{array}$ \\
7 & $\rightarrow$ & 4 \\
\hline 6 & $\rightarrow$ & 4 \\
\hline 5 & $\rightarrow$ & 3 \\
\hline 4 & $\rightarrow$ & 3 \\
\hline 3 & $\rightarrow$ & 2 \\
\hline 2 & $\rightarrow$ & 2 \\
\hline 1 & $\rightarrow$ & 1 \\
\hline 0 & $\rightarrow$ & 1 \\
\hline
\end{tabular}

Figure 5. Priority mapping between IEC 61850 and Ethernet Switches.

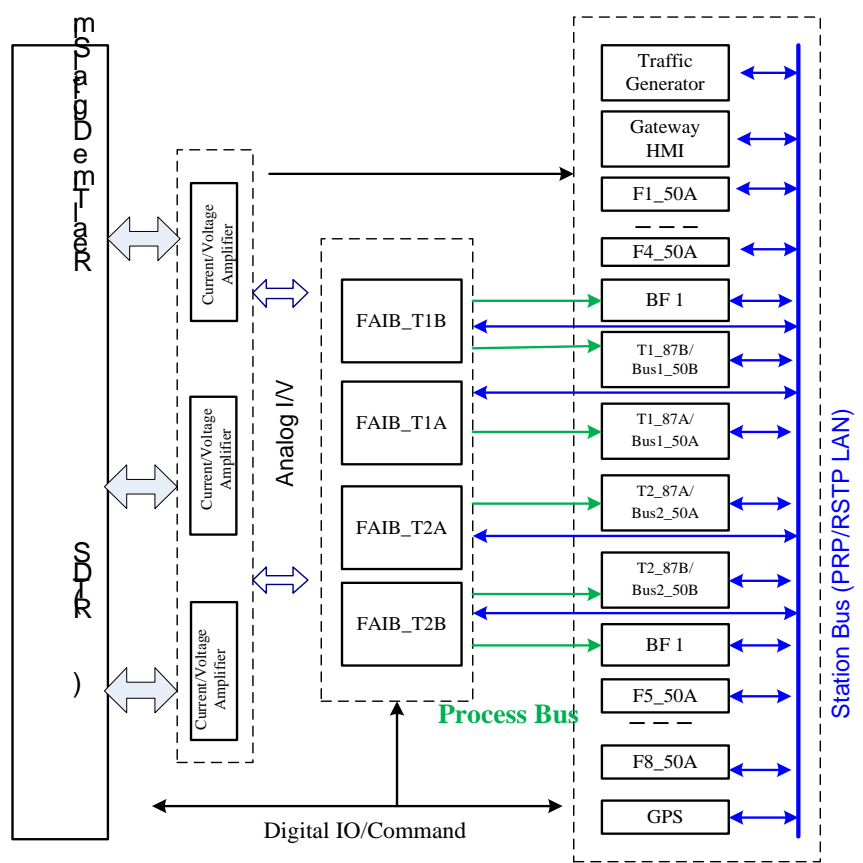

Figure 6. RTDS test setup.

Table 3. Message priority assignments.

\begin{tabular}{cc}
\hline Message & Priority \\
\hline Tripping & 7 \\
BF Tripping & 7 \\
BF Initiation & 7 \\
Reclose Initiation & 5 \\
Reclose Blocking & 5 \\
Open/Close & 5 \\
Switchgear Status & 3 \\
Alarm & 3 \\
Other Network Traffic & Best effort \\
\hline
\end{tabular}


The RTDS simulates the power system normal and fault conditions. Currents and voltage signals at the relaying points are outputted to the amplifiers, which are scaled to the true secondary level and injected on the field apparatus interface units (FAIUs) and indoor switchgear protective relays under testing, and the responses from the feeder relays and field interface units (RAIBs) are fed back into the RTDS, which open/trip the circuit breakers in real time, while the simulation continues, until the power system under study reached a new steady state operational point, then starts the next round simulation. The RTDS dynamic simulation and testing configuration is given in Figure 6, and a RTDS batch test program is developed which enables the test to be repeated as pre-defined. A RTDS batch test program is developed which enables the test to be repeated as pre-defined. A total one hundred (100) tests are configured to verify the performance of the proposed smart substation solutions under various background traffic conditions.

A traffic simulation software developed by the university is hosted in the engineering station, which generates the pre-configured multicasting/unicasting traffic and injects onto the station bus PRP/RSTP LAN, where the secondary system under testing. The multicasting traffic is set with various priority tags, and is intended to flood the LAN under test.

Figure 7 demonstrates the trip times recorded for a Bus1 A-G fault with $0.01 \Omega$ fault resistance. The background traffic injected onto the station bus LAN under each test is given in Table 4.

Analyzing the above test results recorded, the following are observed:

1) Bus 1 relays detect the A-G fault in $12 \mathrm{~ms}$ - $20 \mathrm{~ms}$, with the first relay decision time recoded, and the second discarded.

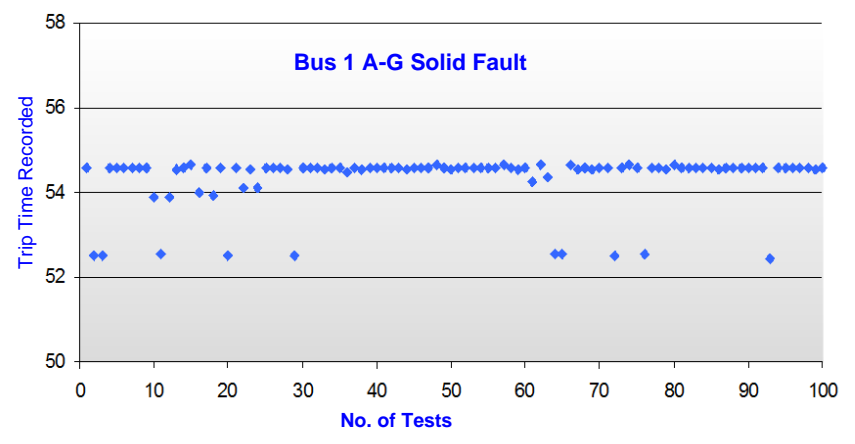

Figure 7. Trip times recorded for 100 RTDS tests.

Table 4. Preconfigure traffic injection.

\begin{tabular}{cccccc}
\hline Test & No. & Multicast & & Unicast & Total \\
\hline Start & End & Volume (mbps) & Priority & mbps & $(\%)$ \\
\hline 1 & 1 & & & & \\
2 & 5 & 10 & 5 & 20 & 30 \\
6 & 10 & 10 & 6 & 20 & 30 \\
11 & 15 & 10 & 7 & 20 & 40 \\
16 & 20 & 15 & 5 & 40 & 45 \\
21 & 25 & 15 & 6 & 40 & 45 \\
26 & 30 & 15 & 7 & 50 & 75 \\
31 & 40 & 25 & 5 & 50 & 75 \\
41 & 50 & 25 & 6 & 50 & 75 \\
51 & 60 & 25 & 5 & 50 & 85 \\
76 & 75 & 35 & 6 & 50 & 85 \\
\hline
\end{tabular}


2) The recoded Bus relays trip decisions are consistent between $52 \mathrm{~ms}$ - $55 \mathrm{~ms}$. This reflects the two (2) cycles $40 \mathrm{~ms}$ preset time delays to ensure blocking signals from the downstream feeder relays are received.

3) The $110 \mathrm{kV}$ circuit breaker opens generally in less than $100 \mathrm{~ms}$, where two cycles' breakers are utilized and simulated in the RTDS.

4) No packagesare dropped off and end point relays. This scenario is not observed throughout the entire batch test duration.

5) Influence of the background traffic injected onto the LAN on relay trip decisions—is not observed.

\section{Conclusion}

This paper investigated into the dynamic simulation testing for evaluation of smart substation solution applied on brown field $110 \mathrm{kV} / 10 \mathrm{kV}$ substations. The RTDS simulated substation under testing in detail, and the rest of the power grid is simplified, where a true substation LAN and process buses are constructed with secondary devices and FAIUs connected. The FAIUs acquire the analog current/voltage signals from RTDS amplifiers, and digitizes them into sampled value streams that multi-casted onto the process bus LAN. Extensive RTDS tests performed in the Smart Substation Technologies Lab, have shown that the smart solution is viable, robust, meets and exceeds the reliablity requirement. It is easy to install and maintain, of incremental upgrade advantages, with performance meets and exceeds the conventional hard wired copper substation solutions.

\section{References}

[1] Song, X.K., Liu, Y., Shen, J., Zhou, G.H., Xiao, Z.H. and Xin, P.Z. (2013) Design of Integrated Information Platform for New Generation Smart Substation. Electric Power Construction, 34, 20-25.

[2] Song, X.K., Shen, J., Li, J.R., Xiao, Z.H., Li, Z.Y., Zou, G.H. and Huang, B.Y. (2013) Design of New Generation Smart Substation. Electric Power Construction, 34, 11-15.

[3] Mo, J. and Tan, J.C. (2014) A Mathematical Model of Process Bus Communication in Smart Substations. Proceedings of the CSEE, 34, 1072-1078.

[4] Xiong, X.P., Tan, J.C. and Lin, X.N. (2012) Reliability Analysis of Communication Systems in Substation Based on Dynamic Fault Tree. Proceedings of the CSEE, 32, 135-141.

[5] Yang, L., Crossley, P.A., Zhao, J.G., et al. (2009) Impact Evaluation of IEC 61850 Process Bus Architecture on Numerical Protection Systems. Proceedings of the 1st International Conference on SUPERGEN, Nanjing, 1-6.

[6] Ingram, D.M.E., Steinhauser, F., Marinescu, C., et al. (2012) Direct Evaluation of IEC 61850-9-2 Process BUS Network Performance. IEEE Transactions on Smart Grid, 3, 1853-1854.

[7] Tournier, J.-C. and Werner, T. (2010) A Quantitative Evaluation of IEC61850 Process Bus Architectures. Proceedings of Power \& Energy Society General Meeting, 1-8.

[8] Kanabar, M. and Sidhu, T. (2009) Reliability and Availability Analysis of IEC 61850 Based Substation Communication Architectures. The 2009 IEEE PES General Meeting, Calgary. 